\title{
Enfoque clínico de niños con síndrome de Down en el consultorio dental
}

\section{Clinical approach of Down syndrome children in the dental office}

\author{
Areias C*, Pereira ML**, Pérez-Mongiovi D*, Macho V*, Coelho A*, Andrade D*, \\ Sampaio-Maia B*
}

\section{RESUMEN}

Introducción: Este estudio pretende describir las características de los niños con síndrome de Down y relacionarlos con la salud oral, con el fin de facilitar el tratamiento en el consultorio dental.

Material y métodos: Fue realizado un estudio de base de datos PubMed limitado a artículos publicados en inglés, español, francés y portugués, entre los años 1997 y 2012.

Resultados y discusión: Necesidad de caracterizar los niveles de salud oral en la población con trisomía 21 con el objetivo de identificar y cuantificar las prioridades en términos preventivos y curativos.

Palabras clave: Síndrome de Down, caries dental, pediatría, saliva.

\section{SUMMARY}

Introduction: This study intends to describe the characteristics of Down syndrome children and relate them to oral health in order to facilitate their treatment in the dental office.

Material and methods: A PubMed database research was conducted and limited to articles published in English, Spanish, French and Portuguese, between the years of 1997 and 2012.

Results and discussion: The characterization of oral health standards in a population with T21 is necessary to establish priorities (both preventive and curative actions) and it's essential to identify and quantify the needs of the target population when a health care plan is made in order to develop a preventive standard protocol.

Key words: Down syndrome, dental caries, pediatrics, saliva.

Fecha de recepción: 6 de diciembre de 2013.

Aceptado para publicación: 5 de abril de 2014 .

* $\quad$ Facultad de Odontología de la Universidad de Oporto. Portugal.

* $\quad$ EPIUnit. Instituto de Salud Pública de la Universidad de Oporto. Portugal.

Areias C, Pereira ML, Pérez-Mangiovi D, Macho V, Coelho A, Andrade D, Sampaio-Maia B. Enfoque clínico de niños con síndrome de Down en el consultorio dental. Av. Odontoestomatol 2014; 30 (6): 307-313.

\section{INTRODUCCIÓN}

\section{Definición y prevalencia}

La trisomía 21 o síndrome de Down es una condición genética que fue descrita por primera vez por John Langdon Down, hace más de un centenar de años $(1,2)$.
Es la anomalía cromosómica más frecuente entre los niños (2-5), con una incidencia de alrededor de 1:770 entre los caucásicos y 1:3.300 a 1:2.000 en la población en general $(1,2-8)$.

El diagnóstico de la trisomía 21 debe ser confirmado con la presencia en el cariotipo de la porción de un fragmento de banda adicional 21q22 (2-5), debido a 
la trisomía libre en la mayoría de los casos, como resultado de la no disyunción meiótica en uno de los padres (5). La causa de este el cambio no se conoce pero puede ocurrir de tres maneras diferentes: la trisomía libre del cromosoma 21, mosaicismo o translocación.

Los niños con trisomía 21 tienen particularidades físicas, mentales y sociales, así que el cuidado de la salud oral debe ser siempre específico y multidisciplinar $(1,2,5)$.

\section{Características orales y maxilofaciales}

Las características orales y maxilofaciales (y, en algunos casos, las condiciones de salud física y mental) presentados por los niños con síndrome de Down son particulares y los cuidados de la salud oral pueden tener que ser adaptados a este grupo (6-10).

En cuanto a las características maxilofaciales, paladar pequeño y estrecho, lengua fisurada, pseudomacroglosia, movimiento lento e incorrecto de la lengua, cierre incompleto del labio, labios hipotónicos, subdesarrollo del maxilar y del tercio medio facial, nariz pequeña, perfil plano o progenie, alta incidencia de bruxismo nocturno, hipotonía, hiperflexibilidad y laxitud del ligamento son muy comunes.

En cuanto a las características dentales, pueden ser encontradas, la implantación irregular de dientes, retención prolongada de dientes deciduos, agenesia dental o anodoncia, dientes supernumerarios, alta frecuencia de mordida abierta anterior y mordida cruzada posterior, forma anormal de los dientes (cónica) con mayor frecuencia en los dientes permanentes, defectos en el esmalte, enfermedad periodontal y baja prevalencia de caries (2,3,5-11).

La erupción de la dentición, tanto en dientes temporales como permanentes, a menudo tarda entre 618 meses y con un patrón típico de erupción, sobre todo en la dentición temporal. En los niños con síndrome de Down, no es habitual que los dientes aparezcan antes de los 9 meses de edad. El primer diente aparece a menudo entre los 12 y 20 meses y la dentición temporal se completa alrededor del cuarto o quinto año de edad (10,11-16). La agenesia dental es una característica frecuente en la dentición permanente, con una frecuencia diez veces mayor que en población general (11).

Por lo tanto, si los factores de riesgo son identificados temprano en la vida de los niños con trisomía 21 (así como en los niños, en general) la pérdida de dientes puede ser reducida o eliminada a través de principios de intervención (17-20). Los efectos de la pérdida de dientes tienen gran relevancia ya que alteran las funciones del sistema estomatognático e interfiere en el crecimiento general y craneofacial, así como en el desarrollo psicológico $(2,3,10,15)$.

El propósito de este estudio es describir las características de los niños con síndrome de Down y relacionarlas con la salud oral, con el fin de facilitar el tratamiento de estos pacientes en el consultorio dental.

\section{MATERIAL Y MÉTODOS}

Fue realizada una investigación en la base de datos PubMed para seleccionar artículos relevantes relacionados con el tema. Las palabras clave fueron: "trisomía 21", "decaimiento", "pediatría", "saliva", "Streptococcus", "Lactobacillus", "Candida" e "iones".

La investigación se limitó a los artículos publicados entre los años 1997 y 2012, en inglés, español, francés y portugués.

\section{RESULTADOS Y DISCUSIÓN}

\section{El síndrome de Down y la salud oral}

\section{La caries dental}

A pesar de la diversidad de resultados encontrados en la literatura, la mayoría de los estudios revelaron que los niños con trisomía 21 tienen una menor prevalencia de caries en comparación con niños no portadores (21-27). Esto se puede explicar por el retraso observado en la erupción de los dientes (618 meses) en ambas denticiones (que conduce a 
menos tiempo de exposición a factores de riesgo) (11) y por el aumento de la capacidad tamponante de la saliva observada en estos niños (24).

Por otra parte, ya que los niños con síndrome de Down visitan al dentista temprano en la vida, esto puede ayudar a alertar a los padres de los factores de riesgo relacionados con la caries dental y las medidas de salud bucal que pueden ser implementadas $(6,7)$.

La existencia de microdoncia y diastema permite no sólo una detección temprana de caries con un simple examen clínico, sin necesidad de un examen radiológico, sino también una limpieza fácil de todas las superficies de los dientes $(5,8,11)$.

El desgaste dental en las superficies oclusales, facilitando la autolimpieza y la higiene oral, la eliminación de alimentos que podrían quedar retenidos en el surco gingival y servir como un sustrato para el desarrollo de caries (6).

\section{Anomalías oclusales}

En los niños con síndrome de Down existe una mayor prevalencia de problemas de ortodoncia con una alta frecuencia de mordida abierta anterior, mordida cruzada posterior, subdesarrollo del maxilar y del tercio medio facial. Macho et al (8) estudiaron anomalías oclusales en pacientes con trisomía 21 y concluyeron que presentaban los siguientes problemas: mordida abierta anterior (54\%), mordida cruzada posterior (97\%), clase III de oclusión (65\%) y overjet mandibular (69\%).

Oredugba (17) encontró $51 \%$ de la clase I y $47 \%$ de la clase III en 43 personas con síndrome de Down vs $5 \%$ de la clase III en el grupo control $(p<0,05)$. Soares et al (18) concluyeron que la clase III es más frecuente en las personas con síndrome de Down. Esto es una consecuencia del hipodesarrollo del tercio medio facial (17). Anatómicamente, el tercio medio facial está subdesarrollado pero la mandíbula sigue un desarrollo normal (pseudoprogenie) (19). Esta displasia del tercio medio facial también contribuye a la estrecha maxilla (19). Oliveira et al (9) encontraron un $31 \%$ de prevalencia de mordida cruzada poste- rior, y Soares et al (18) identificaron mordida cruzada posterior en el $39 \%$ de su muestra.

Ha sido descrito que los niños con trisomía 21 tienen una mayor prevalencia de bruxismo (23\%) en comparación con sus hermanos (2\%) (6). Esta diferencia se puede explicar por la alta prevalencia de anomalías oclusales que se encuentran en los pacientes con síndrome de Down y por la laxitud de las articulaciones y de los ligamentos dentoalveolar y temporomandibular $(2,8)$.

Inicialmente el bruxismo conduce a la erosión de fosas y fisuras, creando superficies oclusales lisas. En los casos en que ya existe el bruxismo, es necesario cambiar la posición de la mandíbula (13).

\section{La enfermedad periodontal}

La enfermedad periodontal es una de las principales causas de la pérdida de dientes en todo el mundo. Es una infección crónica de los tejidos periodontales (encía, ligamento periodontal, cemento radicular y hueso alveolar), debido a la acumulación de placa con una alta prevalencia de bacterias gram negativo (2).

La enfermedad periodontal comienza muy temprano en la vida de los pacientes con síndrome de Down y es la enfermedad bucal más común en estos niños, con un efecto negativo en su calidad de vida $(6,7)$. Las personas con este problema tienen una mala higiene bucal y es común encontrar la acumulación de placa, gingivitis y periodontitis (6).

Hay una alta incidencia de úlceras, candidiasis oral y gingivitis ulceronecrotizante aguda (1). El aumento en la incidencia de la enfermedad periodontal puede ser explicado por la alteración de la función de los leucocitos (responsable de los mecanismos de defensa en los tejidos periodontales), por hipotonía muscular y sus consecuencias, por laxitud articular dentoalveolar, por la falta de comprensión de la importancia de las necesidades de higiene bucal y por la disminución de la destreza manual debido al deterioro cognitivo.

Las personas con síndrome de Down son a menudo afectadas por la destrucción periodontal severa. Un 
comprometido sistema inmune (5), que contiene un número reducido de células $\mathrm{T}$ típicos en la mayoría de los pacientes con trisomía 21 , contribuye a un alto nivel de infecciones y también es uno de los factores que explican la alta incidencia de la enfermedad periodontal. Músculos hipotónicos de la cavidad oral afectan el posicionamiento de la lengua, disminuyendo su eficacia en la deglución y el cambio de la apertura y cierre de la boca (5).

La dificultad para hacer gárgaras y tragar, asociado con una mala masticación afecta la limpieza natural de los dientes, por lo que es importante establecer medidas de higiene oral eficaces. En consecuencia se puede producir halitosis, mal sabor de boca, molestias durante la masticación y pérdida prematura de dientes permanentes. La gingivitis y la enfermedad periodontal comienzan temprano en la vida y su gravedad aumenta con la edad. El 58\% de los pacientes con síndrome de Down tienen periodontitis antes de los 35 años, que es la principal causa de pérdida de dientes en esta población (15).

\section{Microbiología de la saliva}

La evidencia actual muestra que algunas bacterias (Streptococcus mutans, Lactobacillus, Actinomyces) son más importantes que otras en el desarrollo de caries (28-30).

Lenander-Lumikari $M$ et al (31) evaluaron la prevalencia de la necesidad de tratamientos orales (caries y la enfermedad periodontal) en adultos institucionalizados con trisomía 21, comparándolos con un grupo control de la misma edad. Los individuos con trisomía 21 sin caries exhibieron una menor cantidad de Streptococcus mutans y la incidencia de caries en individuos con trisomía 21 fue más pequeña en comparación con el grupo control. Estos resultados corroboran los hallazgos de Areias $\mathrm{C}$ et al $(6,7)$.

En otro estudio en el que los pacientes con trisomía 21 fueron comparados con otras personas con deterioro cognitivo, hubo una menor incidencia de caries entre los individuos con síndrome de Down (11). Los autores atribuyen este hecho a varios factores, como el aumento de la capacidad amortiguadora de la saliva, la tendencia a presentar bruxismo (creación de superficies oclusales lisas) y la anatomía dental peculiar (microdoncia con diastema) (11). La existencia de una diferencia significativa en las diferencias encontradas en los individuos con Streptococcus mutans y Lactobacillus corroboran la literatura actual, que identifica a estos microorganismos como los principales agentes cariogénicos.

Algunos estudios han sugerido una fuerte relación entre la prevalencia de infección por Candida y la caries, especialmente en niños, adolescentes y jóvenes adultos. Law $\mathrm{V}$ et al verificaron un papel activo de C. albicans en la patogénesis de la caries (26). Más recientemente, Areias $\mathrm{C}$ et al demostraron que no había relación entre la caries dental y C. albicans y Lactobacillus (7).

La caries dental tiene una etiología multifactorial. La presencia de altos niveles de Streptococcus mutans y Lactobacillus en la saliva no significa necesariamente que el niño corra un alto riesgo de desarrollar esta enfermedad.

\section{Bioquímica de la saliva}

Además de los factores microbianos, diversos componentes salivales están conectados con la prevalencia de caries $(6,7,30)$.

Varios autores han estudiado el flujo salival, llegando a la conclusión de que es menor en los niños con trisomía 21 (probablemente debido a la postura y medicación) y adicionalmente un menor número de caries dental, en comparación con la datos observados en los grupos control (6,7,32-38).

La existencia de cambios en la función secretora de las glándulas salivares de individuos con trisomía 21 y/o su hipotonía muscular, determina una disminución en el flujo salival (8).

En cuanto el pH de la saliva de individuos con trisomía 21, no hay consenso en la literatura. Hay algunos estudios en los que los valores son superiores (28) en comparación con las personas comunes, mientras que en otros se han observado valores similares $(7,29,38)$ o inferiores $(37)$. Hay varios facto- 
res que podrían influir en los resultados descritos en la literatura, tales como: el método de colecta (como el usado por cada investigador), la edad de los individuos, la ubicación geográfica, los hábitos alimentarios y el tiempo de colecta $(36,37)$. La capacidad tampón de la saliva es la capacidad de prevención de cambios en el pH del entorno (es decir, el sistema tampón es el principal determinante de $\mathrm{pH}$ salival). Lenander-Lumikari $M$ et al (31) estudiaron la variación del pH en los adolescentes y adultos con trisomía 21. Sin embargo, la subjetividad del método que fue utilizado puede haber influido en los resultados.

Siqueira et al (24) estudiaron toda la saliva estimulada y no estimulada de las personas con síndrome de Down 2-5 años y encontraron que la capacidad amortiguadora salival de estos individuos se incrementa en comparación con los individuos sanos de la misma edad.

La amilasa salival es una enzima importante presente en la cavidad oral. Estos autores mostraron baja actividad de la enzima en los individuos con trisomía $(21,37)$. Areias $\mathrm{C}$ et al (7) encontraron diferencias estadísticamente significativas entre la caries dental y la amilasa. La IgA es la inmunoglobulina predominante en la saliva y es producida por las células plasmáticas de las glándulas salivales. La IgA evita la adherencia microbiana, lo que también puede justificar la reducción de la prevalencia de caries en los niños con síndrome de Down (35-38).

Una disminución de los niveles de IgA en niños con trisomía 21 (aunque sin significación estadística) se explica por el inicio de un estado de inmunodeficiencia (7).

Otros estudios han mostrado diferencias relativas a la IgA (mayor en grupo con síndrome de Down) (35), sodio, cloro y calcio (mayor en el grupo con síndrome de Down), potasio (mayor en el grupo de hermanos sin síndrome de Down) (37), amilasa (inferior en el grupo con síndrome de Down) (37).

Siqueira et al (28) mostraron que los individuos con trisomía 21 tienen en la saliva una mayor concentración de proteínas, hecho que puede estar relacionado con el bajo flujo de saliva. Siqueira WL et al $(24,29,30)$ concluyó que los niños con trisomía 21 tienen cambios en los conductos de las células acinares y por lo tanto cambios iónicos (disminución del flujo salival, aumento de la concentración de sodio y potasio y una disminución del $\mathrm{pH}$ ). Otros iones analizados como el cinc, el magnesio, el fósforo y el calcio no mostraron diferencias estadísticamente significativas entre el grupo con síndrome de Down y grupo control $(6,7,32)$.

\section{Primera cita con el dentista pediátrico}

En Portugal, los pacientes con síndrome de Down suelen ser acompañados por un equipo multidisciplinario, incluyendo odontología pediátrica. Por otro lado, los padres se organizan en asociaciones muy activas que transmiten la importancia de la vigilancia de la salud oral a sus miembros desde una edad temprana.

Según Areias C et al $(6,7)$, los niños con trisomía 21 inician la visita al dentista antes que sus hermanos, probablemente debido a una mayor preocupación de los padres. Este hecho también puede explicar la menor tasa de DMF encontrado por algunos autores, teniendo en cuenta que los padres son los primeros alertados de la necesidad de establecer servicios de salud bucal efectivos (21-27). Una cita con el dentista regularmente es importante en todas las edades, pero es esencial en la infancia y adolescencia (24,27-29).

De la información que ha sido recopilada, es posible concluir que los niños con síndrome de Down tienen una baja prevalencia de caries, que puede estar asociada con una gran preocupación de los padres acerca de la salud oral, una pronta visita al dentista (observación clínica, aplicación de flúor y de selladores de fosas y fisuras), presencia de bruxismo, retraso en la erupción, presencia de diastema y un bajo número de Streptococcus mutans en la saliva.

Para la caracterización de los niveles de salud oral en una población es necesario establecer prioridades (acciones preventivas y curativas) y es esencial identificar y cuantificar las necesidades de la población objeto de estudio, con el objetivo de desarrollar un protocolo estándar preventivo. 


\section{BIBLIOGRAFÍA}

1. Shore S, Lightfood T, Ansell P. Oral disease in children with DS: causes and prevention. Community Pract 2010;83(2):18-21.

2. Andrade DJ. Trissomia 21 - Estudo Dento-MaxiloFacial [tesis doctoral]. Porto: FMD-UP; 2000.

3. Moura CP, Vales F, Andrade D, Cunha LM, Barros $\mathrm{H}$, Pueschel SM et al. Rapid maxillary expansion and nasal patency in children with Down syndrome. Rhinology Jun 2005;43(2):138-42.

4. Klug WS, Cummings MR, Spencer CA. Concepts of Genetics. Pearson Education International 2006:191-3.

5. Macho V. Caracterização de uma população pediátrica com Trissomia 21 [master tesis]. Porto: FMD-UP; 2007.

6. Areias C, Sampaio-Maia B, Guimaraes H, Melo P, Andrade D. Caries in portuguese Down syndrome children. Clinics 2011;66(7):1183-6.

7. Areias C, Sampaio-Maia B, Pereira L, Teixeira Á, Andrade D. S mutans and caries in Portuguese Down Syndrome children. Clinics 2012;67(9): 1007-11.

8. Macho V, Seabra M, Pinto A, Soares D, Andrade A. Alterações craniofacial e particularidades orais na Trissomia 21. Acta Pediatr Port 2008;39(5): 190-4.

9. Mouradian WE. The face of a child: children's oral health and dental education. J Dent Educ Sep 2001; 65(9): 821-31.

10. Moura CP. Trissomia 21- Perspectiva Otorrinolaringológica. Arquivos de Medicina 2004.

11. Acerbi AG, Freitas C, Magalhaes MH. Prevalence of numeric anomalies in the permanent dentition of patients with Down syndrome. Spec Care Dentist 2001;21(2):75-8.

12. Allison PJ, Hennequin M, Faulks D. Dental care access among individuals with Down syndrome in France. Spec Care Dentist 2000;20(1):2834.

13. Moreira LM. A síndrome de Down e sua patogénese: considerações sobre o determinismo genético. Rev Bras Psiquiatr 2000;22(2):96-9.

14. Lewis R. Human genetics concepts and applications. 7th ed: McGraw- Hill International Edition; 2007.

15. Paiva E, Ferreira LP. Avaliação do risco de cárie em Odontopediatria: A sua utilidade como meio de prevenção. Acta Pediatr Port 2009;40(2):5964.

16. Holst D, Schuller AA, Aleksejuniene J, Eriksen HM. Caries in populations - a theoretical, causal approach. Eur J Oral Sci 2001;109(3):143-8.

17. Lewis CW, Grossman DC, Domoto PK, Devo RA. The role of the pediatrician in the oral health of children: a national survey. Pediatrics 2000 Dec;106(6):84.

18. Chung MH, Kaste LM, Koerber A, Fadavi S, Punwani I. Dental and medical student's knowledge and opinions of infant oral health. Dent Educ 2006 May;70(5):511-7.

19. Schaff-Blass E, Rozier RG, Chattopadhyay A, Quinonez R, Vann WF Jr. Effectiveness of an educational intervention in oral health for pediatric residents. Ambul Pediatric 2006 May-Jun;6(3): $157-64$

20. Areias C, Macho V, Frias-Bulhosa J, Guimaraes $\mathrm{H}$, Andrade C. Saúde oral em pediatria. Acta Pediatr Port 2008; 39(4): 163-70.

21. Steinberg AD, Zimmerman S. The Lincoln dental caries study: a three year evaluation of dental caries in persons with various mental disorders. J Am Dent Assoc 1978;97(6):981-4.

22. Fung $\mathrm{K}$, Lawrence $\mathrm{H}$. A paired analysis of correlates of dental restorative care in sibling with and without DS. Spec Care Dentist 2008;28(3): 85-91. 
23. Fung K, Allison PJ. A comparison of caries rates in non-institutionalized individuals with and without Down syndrome. Spec Care Dentist 2005;25 (6):302-10.

24. Siqueira WL. Buffer capacity, $\mathrm{pH}$, and flow rate in saliva of children aged 2-60 months with Down syndrome. Clin Oral Investig 2005;9(1):26-9.

25. Tenuta LM. Effect of sucrose on the selection of mutans streptococci and lactobacilli in dental biofilm formedin situ. Caries Res 2006;40(6):546-9.

26. Law V, Seow W, Town G. Factors influencing oral colonization of mutans streptococci in young children. Aust Dent J 2007;52:(2):93-100.

27. Vicente VA. Relação entre a prevalência da doença cárie e risco microbiológico. Cienc Odontol Bras 2008;11(2):44-8.

28. Gizani S. Streptococcus mutans and Streptococcus sanguinis Colonization correlated with caries experience in Children. Caries Res 2008;42:444-8.

29. Siqueira WL, de Oliveira E, Mustacchi Z, Nicolau J. Electrolyte concentrations in saliva of children aged 6-10 years with Down syndrome. Oral Surg Med Pathol Oral Radiol Endod 2004 Jul;98(1):76-9.

30. Siqueira WL, Nicolau J. Stimulated whole saliva components in children with Down syndrome. Spec Care Dentist 2002 Nov-Dec;22(6):226-30.

31. Lenander-Lumikari M, Loimaranta V. Saliva and dental caries. Adv Dent Res Dec 2000;14:40-7.

32. Lee SR, Kwon HK, Song KB, Choi YH. Dental caries and salivar immunoglobulin A in Down syndrome children. J Paediatr Child Health 2004 Sep-Oct;40(9-10):530-3.
33. Cornejo LS, Brunotto M, Hilas E. Salivary factors associated to the prevalence and increase of dental caries in rural schoolchildren. Rev Saude Publica 2008;42(1):19-25.

34. Kavanagha DA, Svehla G. Variation of salivary calcium, phosphate and bufering capacity in adolescents. Arch Oral Biol 1998;43:1023-7.

35. Vijayaprasad KE, Ravichandra KS, Vasa A, Suzan S. Relation of salivar calcium, phosphorus and alkaline phosphatase with the incidence of dental caries in children. J Indian Soc Pedod Prev Dent 2010;28:156-61.

36. Siqueira WL, Siqueira MF, Mustacchi Z, de Oliveira E, Nicolau J. Salivary parameters in infants aged 12 to 60 months with Down syndrome. Spec Care Dentist 2007 Sep-Oct;27 (5):202-5.

37. Davidovich E, Aframian DJ, Shapira J, Peretz B. A comparison of the sialochemistry, oral $\mathrm{pH}$, and oral health status of Down syndrome children to healthy children. Int J Paediatr Dent Jul 2010; 20 (4):235-41.

38. Areias C, Sampaio-Maia B, Macho V, Leal I, Melo $P$, de Andrade $C$. Does the chemistry in the saliva of Down syndrome children explain their low caries prevalence? Eur J Paediatr Dent Mar 2013; 14(1):23-6.

\section{CORRESPONDENCIA}

Cristina Areias

Rua Amadores Teatrais fangueiros, 92

4740-343 Fão - Portugal

E-mail: careias@fmd.up.pt 